The first three chapters discuss the relationship of nutrition to disorders of the haematopoietic, nervous and musculoskeletal systems respectively. These chapters are not exhaustive and are not concerned with therapeutic aspects. The effects of essential nutrients on the gastrointestinal tract and the effects of diseases of the tract on the absorption and utilization of nutrients are dealt with, as also are the nutritional abnormalities occurring in liver disease. Here again there is no discussion of the possible role of nutrition in treatment. This is in contrast to the presentation on cardiac failure which is much concerned with therapeutic aspects and contains a useful table of drug-nutrient interactions of importance in congestive heart failure.

Discussion of the relationship of diet and nutrition to cancer is mainly concerned with their possible involvement in the aetiology of oncological disease, with little mention of the effects of the disease on the nutrition of the patient.

The ageing-nutrition-health triad is discussed by Watkins who points out that education is the prime catalyst for inducing change. The chapter on the endocrine system is concerned with diabetes, vitamin D, and parathyroid and thyroid disorders. Discussion of megavitamins and food fads is largely taken up with doubts about Pauling's well-known views. It is, perhaps, unfortunate that in a text of this sort some consideration is not given to the possibility that larger amounts of vitamins than the currently recommended allowances may have beneficial effects which are difficult to measure and therefore of ten dismissed as anecdotal.

There is a useful and well-referenced chapter on the effects of ethanol. Nutritional aspects of infectious diseases

\section{Abdication of reason} John C. Marshall

The Right Brain: A New Understanding of the Unconscious Mind and its Creative Powers. By T. R. Blakeslee. Pp.275. (Papermac/Doubleday: 1980.) Hardback $£ 10, \$ 10.95$; paperback $£ 2.95$.

THIS is another "popular" book on how to unlock the emotional and creative power of the right cerebral hemisphere. The volume recounts some of the evidence, from both normal and brain-injured people, that has led physicians and clinical psychologists to believe that considerable specialization of function characterizes different areas of the human brain. Blakeslee concentrates on inter- rather than intra-hemispheric localization and leaps somewhat wildly from datum to conclusion. For any reviewer who wants to dip into his repertoire of sarcasm, the volume will provide more than adequate stimulus why is there no woman Beethoven, the author asks, and answers that the cerebral are discussed and it is pointed out that nutritional supportive therapy should be employed to prevent or minimize the depletion of body stores during an infection, and to replace lost nutrients as expeditiously as possible during convalescence. Obesity is the most common nutritional disease in Western society. Assessment, risks and treatment of this poorly understood condition are considered. As a consequence of our poor understanding successful treatment is often elusive, and the demand for treatment has led to a multiplication of fad diets, examples of which are given. The final chapter contains a discussion of the interrelationships of nutrition and the kidney. Effects on kidney function and nutritional disturbances in the nephrotic syndrome, hypertension and renal failure are dealt with, as also are the principles of nutritional therapy.

These volumes are a useful addition to the textbooks available on human nutrition. They are not exhaustive, either in respect of subjects covered or the depth to which each subject is discussed, but the material is well presented and well referenced throughout. Moreover, the books have been produced to a high standard and are a pleasure to read. In spite of their inevitably high price, teachers of nutrition at degree level may well consider it worthwhile acquiring one or more of them. They can be said to have achieved their aim and to present in reasonable compass the present state of the art in a dynamic subject.

J.W.T. Dickerson is Professor of Human Nutrition at the University of Surrey and Consultant Adviser on Clinical Nutrition to the South West Thames Regional Health Authority.

hemispheres of women are functionally less differentiated than those of men. It is tempting to regard Blakeslee's offering as beyond satire and plunge immediately into instant sociology. Why do such books exist? What need do they satisfy?

The "right brain revolution" that Blakeslee and so many others have attempted to explain to a lay audience had its genesis in 1962. Two Los Angeles neurosurgeons, Philip Vogel and Joseph Bogen, revived and refined a surgical procedure for the control of pharmacologically intractable epilepsy. The operation involved sectioning the corpus callosum and the anterior and hippocampal commissures - the main fibre tracts that interconnect the two cerebral hemispheres at the cortical level. There can be little doubt that both the original and subsequent series of commissurotomy operations have been a success; patients whose intellectual and social activity had been severely compromised by generalized convulsions have been able to return to an essentially normal life, although still subject, of course, to the disabilities consequent upon their pre-operative brain damage.

Here we also had a neurosurgical preparation in which, with appropriate techniques that were lacking in the $1940 \mathrm{~s}$, one could investigate the cognitive capacities of the left and right hemispheres isolated from each other except through their brain stem connections. The preparation enabled workers to confirm, extend and fine the deductions that had previously been drawn from the effects of lateralized injury, namely, that the left and right hemispheres (in the majority of the population) are indeed primarily implicated in the exercise of linguistic and visuo-spatial skills, respectively. But because in the commissurotomy patient the left hemisphere has little knowledge of what the right hemisphere is doing (and vice-versa), the enticement of talking about these people as if they had two minds within a single body (brain? person? soul?) proved irresistible (and not only to popularizers). The lure of thinking about the patients in this way is, of course, much more exciting than patiently trying to comprehend the what, how and why of functional localization.

But worse was to come. The disconnected right hemisphere, conspicuously deficient in syntax and verbal reasoning, can yet support dreams, emotions, and a high level of non-verbal memory and intelligence. All that remained was to deify the distinction between the cerebral hemispheres: the left brain became the seat of a cold, calculating, logical and talkative mind, whilst the right brain sustained a warm, intuitive, emotional and artistic mind. In an era in which attitudes towards science are often ambivalent, what could be more attractive than to have science and surgery discover the reality, nay the material substrate even, of intuition, creativity and pure feeling.

Modern times are truly an ideal medium in which to culture the hundreds of articles and books that have attempted to disseminate "the right brain revolution" that Blakeslee describes. Yet there is a sense in which any sociological analysis provides too easy a let-out. Blaming the Zeitgeist never takes us very far. The real vacuum that such books attempt to fill arises from the failure of neuroscientists themselves to provide any significant theoretical account of functional localization. The evidence from anatomy and pathology, and from recordings of the electrical and chemical activity of the normal intact brain - now overwhelmingly supports the notion that distinct cerebral regions are differentially involved in the exercise of higher cognitive functions. How and why this is the case we do not know. It could, however, be an interesting problem for someone to work on.

John C. Marshall is in the Neuropsychology Unit, Department of Clinical Neurology, The Radcliffe Infirmary, Oxford. 